\title{
SINGULAR ASYMPTOTICS APPROACH TO PARTIAL DIFFERENTIAL EQUATIONS WITH ISOLATED SINGULARITIES IN THE COEFFICIENTS
}

\author{
BY CONSTANTINE J. CALLIAS ${ }^{1}$ AND GUNTHER A. UHLMANN ${ }^{2}$
}

1. Introduction. In this note we announce some results on the heat equation (§3) and scattering theory (§4) associated with some differential operators with isolated singularities in the coefficients. The results are based on recent joint work by the authors $[\mathrm{CU}]$ and on independent work by the first author $[\mathrm{C1}, \mathrm{C2}, \mathrm{C3}]$. We study the small time asymptotics of the heat kernel and the large frequency asymptotics of the scattering amplitude. These questions are classical but need reinterpretation because of the singularities. Our general approach is to solve the differential equation in question away from the singularity and then try to extend it across. In all of our problems, we found that we can handle the situation by using a singular asymptotics lemma in $D^{\prime}(\mathbf{R})(\S 2)$ for functions of the form $f(s / x, x)$ as $s \rightarrow 0$ with certain quite general conditions on $f$. We believe that the latter result is of general interest and wide applicability $[\mathrm{C1}, \mathrm{C2}, \mathrm{C3}, \mathrm{CM}, \mathrm{CU}, \mathrm{U}]$.

2. Singular asymptotics. (This is based on [CU].)

THEOREM 2.1. Let $f(y, x) \in C^{\infty}((0, \infty) \times \mathbf{R})$ be such that there exist functions $f_{n j}(x) \in C^{\infty}(\mathbf{R})$, a sequence of complex $k_{n}$, $\operatorname{Re} k_{n} \uparrow \infty$, and nonnegative integers $l_{n}$ such that

$$
\left|\partial_{x}^{k} f(y, x)-\sum_{n=0}^{N} \sum_{j=0}^{l_{n}}\left(\partial_{x}^{k} f_{n j}(x)\right) y^{k_{n}}(\ln y)^{j}\right| \leq C_{\epsilon, k, B} y^{\mathrm{Re} k_{N+1}-\epsilon}
$$

as $y \rightarrow 0^{+}$for all $k, N, \epsilon>0$, and for all $|x| \leq B$. Assume further that

$$
\left|\partial_{x}^{k} f(y, x)\right| \leq c_{k} y^{k} g_{k}(y)
$$

for all $x$ in a neighborhood of $0, y \rightarrow \infty$, where $\int_{0}^{c} g_{k}(1 / x) d x<\infty$ for some $c>0$. Let $\omega \in \mathbf{C}, \operatorname{Re} \omega<0$. Then for all $\phi \in C_{0}^{\infty}(\mathbf{R})$ we have an asymptotic expansion as $s \rightarrow 0^{+}$:

Received by the editors April 11, 1983 and, in revised form, December 2, 1983.

1980 Mathematics Subject Classification. Primary 35P25, 35K05, 41A60, 58G99; Secondary 35A30, 35L05, 35R05.

${ }^{1}$ Research partly supported by NSF Grant MCS 82-03972.

${ }^{2}$ Research partly supported by NSF Grant MCS 81-02498.

(C) 1984 American Mathematical Society $0273-0979 / 84 \$ 1.00+\$ .25$ per page 


$$
\begin{aligned}
\int_{0}^{\infty} d x f\left(\frac{s}{x}, x\right) x^{-\omega} \phi(x) \sim & \sum_{\substack{n=0 \\
a=k_{n} \\
k_{n}+\omega \neq m+1 \in N}}^{\infty} \sum_{j=0}^{l_{n}} s^{a}(\ln s)^{j}\left(u_{a, j}(\omega), \phi\right) \\
+ & \sum_{\substack{n=0 \\
a=k_{n} \\
k_{n}+\omega=m+1 \in N}}^{\infty} \sum_{j=0}^{l_{n}+1} s^{a}(\ln s)^{j+1}\left(v_{a, j}(\omega), \phi\right)
\end{aligned}
$$

where $u_{a, j}(\omega), v_{a, j}(\omega) \in D^{\prime}(-\infty, \infty), \operatorname{Re} \omega<0$. Moreover,

$$
\text { sing supp } u_{a, j}(\omega) \subseteq\{0\} \text { and } \operatorname{supp} v_{a, j}(\omega) \subseteq\{0\} .
$$

Note that (2.1) implies an obvious asymptotic expansion of

$$
\int_{0}^{\infty} f\left(\frac{s}{x}, x\right) \phi(x) d x
$$

for $\phi \in C_{0}^{\infty}(0, \infty)$ as $s \rightarrow 0^{+}$. The theorem gives an extension of the expansion for $\phi \in C_{0}^{\infty}(-\infty, \infty)$. We can also get explicit formulas for $u_{a, j}(\omega), v_{a, j}(\omega)$.

The theorem can be used in $\mathbf{R}^{n}$ by using polar coordinates and treating the angular coordinates as parameters. The theorem generalizes various results of the authors (see $[\mathbf{C 1}, \mathbf{C 2}, \mathbf{C 3}, \mathbf{U}]$ ) and is closely related to a recent result of Seeley [S], obtained independently. The proof is very classical in spirit and uses fundamentally the Mellin transform and its properties.

3. Asymptotics of the heat kernel. The heat equation $\left(\partial_{t}+H\right) \psi_{t}=0$, associated with a positive elliptic differential operator $H$, is a powerful tool in the study of spectral properties of such operators. The heat operator is an operator solution subject to $\lim _{t \rightarrow 0} \psi_{t}=I$ and is usually represented by the so-called heat kernel. In this section we give a reinterpretation of the classical small-time asymptotic expansion [G] of the diagonal of the heat kernel for some elliptic differential operators with a finite number of isolated singularities in the coefficients, and we outline an existence proof for the expansion (Theorem 3.1). The results below are based on [C1], which gives the basic ideas in the context of a one-dimensional example, [C2] and [C3].

A typical operator from our class is the Schrödinger operator $H$ on $\mathbf{R}^{n}$ with coefficients singular only on a finite set $S=\left\{y_{1}, \ldots, y_{m}\right\} \subset \mathbf{R}^{n}$ [C2]. Let $U_{1}, \ldots, U_{m}$ be open, $\mathbf{R}^{n}=\bigcup_{j=1}^{m} U_{j}, y_{j} \in U_{j}, y_{i \neq j} \notin U_{j}$. Then $H$, assumed formally selfadjoint, is of the form

$$
H=-\Delta+\sum_{k=1}^{n} b_{k}(x) i \partial_{k}+b(x)
$$

where $b_{k}(x), b(x) \in C^{\infty}\left(\mathbf{R}^{n} \backslash S\right)$ are compactly supported, and, for $x \in U_{j}$, of the form

$$
\frac{1}{\left|x-y_{j}\right|} \psi_{j, k}\left(\frac{x-y_{j}}{\left|x-y_{j}\right|}, x\right), \quad \frac{1}{\left|x-y_{j}\right|^{2}} \psi_{j}\left(\frac{x-y}{\left|x-y_{j}\right|}, x\right),
$$

respectively, for some $\psi_{j, k}(y, x), \psi_{j}(y, x) \in C^{\infty}\left(S^{n-1} \times U_{j}\right)$. Results have been obtained for (3.1) and are expected to apply to analogous operators on manifolds. 
Under suitable conditions on the coefficients of $H$, roughly speaking if $b(x)$ is not too unbounded from below, $H$ will be bounded from below on $C_{0}^{\infty}\left(\mathbf{R}^{n} \backslash S\right)$. By strengthening the conditions, $H$ can be made essentially selfadjoint on $C_{0}^{\infty}\left(\mathbf{R}^{n} \backslash S\right)$. For example, if $b_{k}(x)=0, n \geq 4, H$ is essentially selfadjoint if $b(x) \geq 0$ in a neighborhood of $S$. In our work we assume that $H$ is essentially selfadjoint, although we do not consider this restriction fundamental from the point of view of both the technicalities and the results. The heat operator is now constructed by the spectral theorem as $e^{-t H}$, and we can show that $\phi e^{-t H}$ is Hilbert-Schmidt for $\phi \in C_{0}^{\infty}\left(\mathbf{R}^{n}\right)$ and $e^{-t H}$ has a kernel function $e^{-t H}(x, y)$ which is $C^{\infty}$ for $x, y \in \mathbf{R}^{n} \backslash S$.

We now want to study

$$
\operatorname{Tr} e^{-t H} \phi=\int d x \phi(x) e^{-t H}(x, x)
$$

as $t \rightarrow 0^{+}$to $O\left(t^{\infty}\right)$, which is equivalent to the asymptotics of $e^{-t H}(x, x)$ as $t \rightarrow 0^{+}$in the sense of distributions. (See [C-T, C1] for discussion and applications.) The main result which makes it possible to derive the theorem below from the singular asymptotics lemma is that $e^{-t H}(x, x)$ can be written as a function of the form

$$
F_{j}\left(t, \frac{t}{\left|x-y_{j}\right|^{2}}, x, \frac{x-y_{j}}{\left|x-y_{j}\right|}\right)
$$

for $x$ near $y_{j}$ where $F_{j}(t, \tau, x, y) \in C^{\infty}\left(\mathbf{R}^{+} \times \mathbf{R}^{+} \times \mathbf{R}^{n} \times S^{n-1}\right)$ for each $j$. This statement about the behavior of the heat kernel is of course of interest in itself; it is motivated by looking at small $t$ behavior for $x \notin S$, which is classical, and is proved by a good deal of operator theoretic estimating. Using Theorem 2.1 we obtain

Theorem 3.1. We have as $t \rightarrow 0^{+}$, for each $\phi \in C_{0}^{\infty}\left(\mathbf{R}^{n}\right)$,

$$
\begin{aligned}
\int_{\mathbf{R}^{n}} & e^{-t H}(x, x) \phi(x) d x \\
& \sim t^{-n / 2}\left[\sum_{k \geq 0}^{\infty} u_{k}(\phi) t^{k}+\sum_{k \geq(n-1) / 2} t^{k}\left(v_{k}(\phi) t^{1 / 2}+w_{k}(\phi) \ln t\right)\right],
\end{aligned}
$$

where $u_{k}, u_{k}, w_{k} \in D^{\prime}\left(\mathbf{R}^{n}\right)$, for $k>0$ sing $\operatorname{supp}\left(u_{k}, v_{k}, w_{k}\right)=S$ and $v_{k}, w_{k}$ are combinations of Dirac distributions at $y_{1}, \ldots, y_{m}$.

A similar analysis will apply if we assume that near a singularity, say $x=0$, $b(x)$ has an asymptotic expansion

$$
b(x) \sim \sum_{n=0}^{\infty} \sum_{j=0}^{l_{n}}|x|^{k_{n}}(\ln |x|)^{j} \psi_{n j}\left(\frac{x}{|x|}\right),
$$

where $k_{0}=-2, k_{n} \uparrow \infty, l_{n} \geq 0$ are integers, $\psi_{n j}$ are smooth. In Theorem 3.1 we would obtain more general powers of $t$ and powers of logarithms.

Theorem 3.1 is purely an existence result and cannot be used to determine a computational scheme for the distributional coefficients. A method of calculation has been devised [C3] which again relies on Theorem 2.1. 
4. Scattering by a singular potential. (This is based on [CU].) We study scattering by singular potentials with isolated singularities at the origin using wave equation methods. We describe our result, for simplicity, for a potential with the singularity of the Coulomb potential in three dimensions:

$$
V(x)=b(x) /|x|, \quad b \in C_{0}^{\infty}\left(\mathbf{R}^{3}\right) .
$$

An incoming plane wave with direction $\theta$ and frequency $\lambda$ interacts with the potential. The amplitude of the scattered wave in the direciton $\omega$ is, roughly speaking, the scattering amplitude $a(\lambda, \theta, \omega), \lambda \in \mathbf{R}, \theta, \omega \in S^{2}$. We prove that the scattering amplitude at forward peak $a(\lambda, \theta, \theta)$ has a full asymptotic expansion for large frequencies. From this expansion one can determine the full singularity of the potential at the origin.

THEOREM 4.1.

$$
a(\lambda, \theta, \theta) \sim \sum_{j \geq 0} \lambda^{1-j} u_{j}+\sum_{j \geq 0} \lambda^{2-j} \ln \lambda v_{j}
$$

$\lambda \gg 0$, where $u_{j}, v_{j} \in C^{\infty}\left(S^{2}\right)$. From the $v_{j}$ 's we get $\partial_{x}^{\alpha} b(0)$ for all $\alpha$.

In the case of a smooth compactly supported potential, no log terms appear in the expansion (see $[\mathbf{M}]$ ). The log terms come from the singularity of the potential, and one can get extra information in that case, namely, the singularity of the potential.

We now explain briefly how Theorem 2.1 is used in the proof of Theorem 4.1. We solve the wave equation plus potential with data a plane wave in the far past:

$$
\square_{V} u=\left(\frac{\partial^{2}}{\partial t^{2}}-\Delta+V\right) \mu=0\left(\bmod C^{\infty}\right), \quad \mu=\delta(t-x \omega) t \ll 0, \omega \in S^{2} .
$$

We write a solution of (4.2) as an oscillatory integral with distribution-valued classical symbols:

$$
\mu=\int e^{i\left(t-x_{1}\right) \xi} a(x, \xi) d \xi
$$

where

$$
(a(x, \xi), \phi) \sim \sum_{j \leq 0}\left(a_{j}(x), \phi\right) \xi^{j} \quad \forall \phi \in C_{0}^{\infty}\left(\mathbf{R}^{3}\right), a_{j}(x) \in D^{\prime}\left(\mathbf{R}^{3}\right) .
$$

Using the standard methods of geometrical optics (see $[\mathbf{L}]$ ) we get

$$
a_{0}(x)=1, \quad a_{-1}(x)=\int_{-\infty}^{x_{1}} \frac{b\left(s, x^{\prime}\right)}{\left(s^{2}+\left|x^{\prime}\right|^{2}\right)^{1 / 2}} d s .
$$

$x=\left(x_{1}, x^{\prime}\right) \in \mathbf{R} \times \mathbf{R}^{2}$ (here $\left.x_{1}=x \omega, x^{\prime}=x-(x \omega) \omega\right)$. We write

$$
a_{-1}(x)=\int_{-\infty}^{0} \frac{b\left(s, x^{\prime}\right)}{\left.\left(s^{2}+\left|x^{\prime}\right|^{2}\right)^{1 / 2}\right)} d s+f_{1}\left(\frac{x_{1}}{\left|x^{\prime}\right|},\left|x^{\prime}\right|, \frac{x^{\prime}}{\left|x^{\prime}\right|}\right)
$$

with

$$
f_{1}\left(x_{1},\left|x^{\prime}\right|, \frac{x^{\prime}}{\left|x^{\prime}\right|}\right)=\int_{0}^{x_{1}} \frac{b\left(s\left|x^{\prime}\right|, x^{\prime}\right)}{\left(1+s^{2}\right)^{1 / 2}} d s .
$$


Clearly, $f_{1}\left(x_{1}, R, \theta\right) \in C^{\infty}\left(\mathbf{R} \times \overline{\mathbf{R}^{+}} \times S^{2}\right)$, where $R=\left|x^{\prime}\right|, \theta=x^{\prime} /\left|x^{\prime}\right|$. A similar statement is valid for $a_{-j}(x), j>1$, with $f_{1}$ replaced by $f_{j}$.

To obtain Theorem 4.1 we need to expand $f_{j}\left(x_{1},\left|x^{\prime}\right|, x^{\prime} /\left|x^{\prime}\right|\right)$ asymptotically as $x_{1} \rightarrow 0$ with coefficients in distributions in $x^{\prime}$. This is provided by Theorem 2.1. The proof of Theorem 4.1 follows now, more or less, the lines of [M], using the extension of the Lax-Phillips theory to short range potentials developed by Phillips [Ph].

\section{REFERENCES}

[C1] C. Callias, The heat equation with singular coefficients. I, Comm. Math. Phys. 88 (1983), 357-385.

[C2] _ Asymptotics of the heat kernel for Schrödinger operators with isolated singularities in the coefficients, 1982 (preprint).

[C3] _ The resolvent and the heat kernel for some singular boundary problems, 1983 (preprint).

[CM] C. Callias and X. Markenscoff, Singular asymptotics of integrals and the near field radaited from a non-uniformly moving dislocation, Ninth U. S. Congress of Applied Mechanics, Cornell Univ., June 1982.

[CT] C. Callias and C. Taubes, Functional determinants in Euclidean Yang-Mills theory, Comm. Math. Phys. 77 (1980), 229-250.

[Gr] P. Greiner, An asymptotic expansion for the heat equation, Global Analysis, Proc. Sympos. Pure Math., vol. 14, Amer. Math. Soc., Providence, R. I., 1968, pp. 137-146; Arch. Rational Mech. Anal. 41 (1971), 163-218.

[L] P. D. Lax, Asymptotic solutions of oscillatory initial valued problems, Duke Math. J. 24 (1957), 627-646.

[M] R. B. Melrose, Lectures on scattering theory, M.I.T., 1981.

[Ph] R. Phillips, Scattering theory for the wave equation with a short range perturbation, Indiana Univ. Math. J. 31 (1982), 609-639.

[RS] M. Reed and B. Simon, Methods of modern mathematical physics, Vol. 11, Academic Press, New York, 1975.

[S] R. Seeley, Asymptotics of certain integrals of symbols, 1983 (preprint).

[U] G. Uhlmann, Light intensity distribution in conical refraction, Comm. Pure Appl. Math. 35 (1982), 69-80.

Department of Mathematics, Columbia University, New York, New York 10027 (Current address of C. Callias)

Department of Mathematics, Massachusetts Institute of Technology, Cambridge, Massachusetts 02139 (Current address of G. Uhlmann)

Mathematical Sciences Research Institute, Berkeley, California 94720 